\title{
Ajustement expérimental de la formule de Caquot en hydrologie urbaine
}

par D. Normand

SOGREAH

Les débits de ruissellement d'eaú pluviale à prendre en compte pour calibrer les ouvrages d'assainissement sont, actuellement, déterminés en France, par la formule élaborée en 1941 par M. Caquot, et reprise dans linstruction technique C.G. 1333 de 1949.

Pour préparer la révision de cette instruction ministérielle, actuellement en cours au sein d'une commission présidée par M. Loriferne, le Ministère de l'Equipement nous avait demandé d'efiectuer une étude expérimentale d'hydrologie urbaine qui s'est déroulée de 1968 à 1970. Les conclusions de cette étude, complétées par les résultats d'une recherche confiée au Laboratoire d'Hydrologie de Montpellier, servent actuellement de base aux travaux de la commission Loriferne. Nous ne pouvons donc présenter ici qu'une synthèse provisoire de ces recherches.

\section{1 - Présentation de la formule de Caquot}

Au départ de la démarche qui conduit à la formule de Caquot, on trouve la notion du «temps de concentration $»$, $t_{c}$, défini comme temps de parcours de l'eau de pluie tombée au point extrême du bassin versant pour parvenir à l'exutoire en coulant dans un réseau ramifié de canalisations à plein débit.

Des raisonnements d'ordre hydraulique peuvent conduire à relier $t_{c}$ aux caractères physiques du bassin par une équation du type :

$$
t_{c}=\mu P^{o} S^{d l} Q^{f}
$$

$P=$ Pente moyenne du bassin;

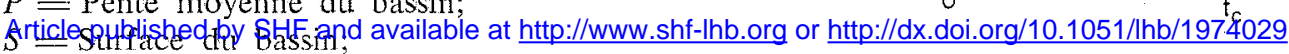
$Q=$ débit maximal à l'exutoire.
Ayant défini $t_{c}$, on ne prend en compte au sein de la pluie que la partie la plus intense de durée égale au temps de concentration; l'intensité moyenne de cette partie intense de l'averse est $I_{m}$.

On admet en outre que l'hydrogramme $q(t)$ résultant de cette averse a son débit maximal $Q$ à la fin de la pluie de durée $t_{c}$ considérée (fig. 1).

On peut alors distinguer dans cet hydrogramme:

- un volume $V 1$ correspondant à l'écoulement lors de la phase de montée du débit;

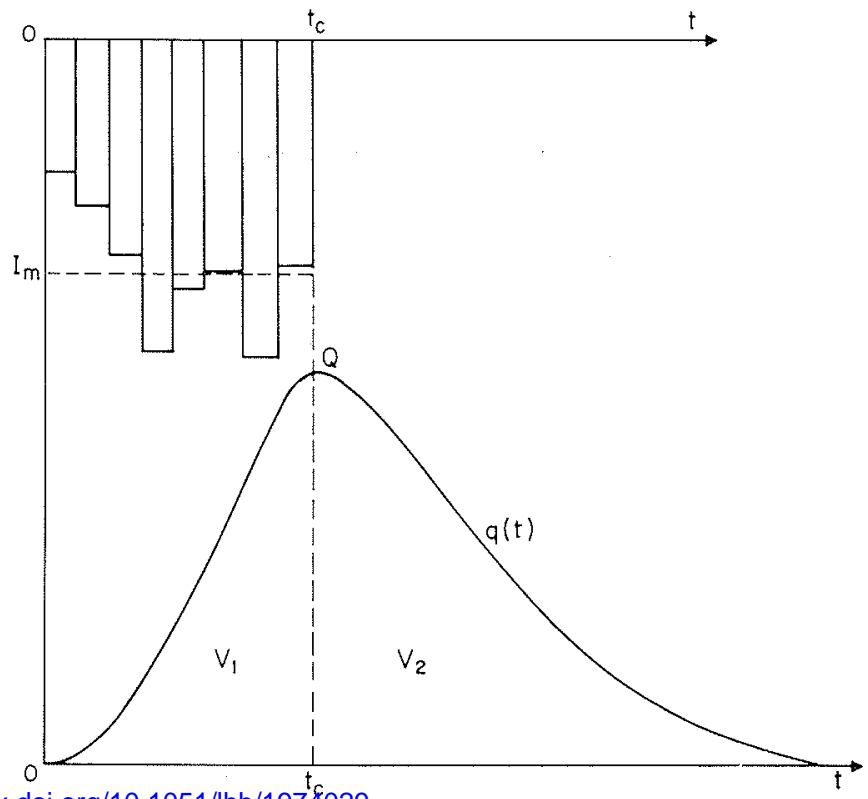


- un volume $V 2$ qui s'écoule lors de la phase de décroissance du débit et qui représente le volume d'eau accumulé momentanément sur le bassin versant et dans les collecteurs.

En posant :

$$
\begin{aligned}
& V 1=\beta Q t_{c} \\
& V 2=\delta Q t_{c}
\end{aligned}
$$

on peut écrire la relation volumétrique simple:

$$
C . I_{m} \cdot t_{c} \cdot S=6 \cdot(\beta+\delta) Q t_{c}
$$

$C=$ coefficient de ruissellement (égal pratiquement au coefficient d'imperméabilisation);

6 = coefficient d'homogénéisation des unités lorsque

$t_{c}$. est exprimé en minutes;

$Q$ est exprimé en $\mathrm{m}^{3} / \mathrm{s}$;

$S$ est exprimé en ha;

$l_{m}$ est exprimé en $\mathrm{mm} / \mathrm{mn}$.

L'intensité $I_{m}$ peut s'exprimer de façon convenable en fonction de $t_{c}$ et de la surface $S$ du bassin par la relation :

$$
I_{m}=a t_{c}^{b} \cdot S^{-\varepsilon}
$$

$I=a t^{b}$ étant la relation intensité durée de la pluie ponctuelle de fréquence considérée;

$S^{-\varepsilon}$ correspondant à l'atténulation de la pluie en fonction de la surface d'impluvium.

En éliminant $I_{m}$ et $t_{c}$ entre les équations (1), (2), (3), on obtient la formule de Caquot:

$Q=\left(\frac{a \mu b}{6(\beta+\delta)}\right)^{\frac{1}{1-b f} \cdot P^{-\frac{b c}{1-b f}} \cdot C^{\frac{1}{1-b f}} \cdot S^{\frac{b d-\varepsilon+1}{1-b f}}}$

Dans cette relation:

$a, b, \varepsilon$ sont des paramc̀tres pluviométriques régionaux;

$\mu,(\beta+\delta), f, c, d$, sont des constantes;

$C, P, S$ caractérisent le bassin versant.

\section{2 - Paramètres pluviométriques}

\section{1 - $a$ ef $b$}

L'intensité moyenne maximale de pluie de fréquence $F$ décroît en fonction de la durée $t$ suivant une relation de la forme :

$$
I_{F}(t)=u(t+w)^{v}
$$

Dans l'élaboration de la formule de Caquot, on simplifie cette relation en :

$$
I_{F^{\prime}}(t)=a t^{\delta}
$$

Cette simplification est légitime si on ne s'intéresse qu'à un intervalle de temps réduit :

$$
6 \mathrm{mn}<t<1 \mathrm{~h} \text { par exemple. }
$$

Les paramètres $a$ et $b$ dépendent de la région considérée et de la fréquence prise en compte. $2.2-\varepsilon$

Lorsque l'on étudie l'atténuation des intensités de pluie en fonction de la surface réceptrice, on est conduit à distinguer des courbes spécifiques à chaque durée (fig. 2).

Il n'est pas illogique de faire passer, dans ce réseau de courbes, une droite $\alpha=S^{-\varepsilon}$ qui coupe chaque courbe $\alpha=(S, t)$ en un point $(S, t)$ tel que $t$ respecte l'ordre de grandeur du temps de concentration d'un bassin de superficie $S$.

Une étude du phénomène «averse » dans laquelle nous avions dissocié :

- l'analyse des hyétogrammes ponctuels;

- l'analyse des décroissances d'intensités maximales en fonction de la distance;

- et les décalages dans le temps de ces intensités maximales; avait conduit à :

$$
\varepsilon=0,015
$$

Cette valeur constitue une limite extrême de $\varepsilon$ puisqua nous faisions alors abstraction des décalages dans le temps entre les hyétogrammes de deux postes pluviométriques.

L'analyse des études de ce type, qui ont été faites dans d'autres pays, montre que la valeur $\varepsilon=0,1$, prise en compte dans la circulaire 1333, constitue cependant une limite supérieure, et qu'un ordre de grandeur raisonnable pourrait se situer aux alentours de $\varepsilon=0,05$.

Comme il est intéressant de mesurer l'incidence pratique du choix de $\varepsilon$ sur l'ajustement final, nous encadrerons la valeur 0,05 par les valeurs extrêmes 0,015 et 0,1 .

\section{3 - Ajustement des constantes}

\section{1 - Méthode " conceptuelle"}

Si l'on admet toutes les hypothèses qui conduisent à la formule de Caquot, telles que nous les avons indiquées en 1 , on peut définir la valeur de chaque constante.

C'est ce qui a été fait par $M$. Caquot et repris par la circulaire CG 1333 , conduisant à :

$$
\begin{array}{ll}
\mu & =0,93 \\
c & =-0,363 \\
d & =0,366 \\
f & =-0,2
\end{array}
$$

Le Laboratoire d'Hydrologie de Montpellier, reprenant ce type d'ajustement à partir d'une analyse de résultats expérimentaux, est conduit à proposer les valeurs suivantes :

$$
\begin{array}{ll}
\mu . & =0,65 \\
c & =-0,41 \\
d & =0,507 \\
f & =-0,287 \\
\beta+\delta & =1,1
\end{array}
$$




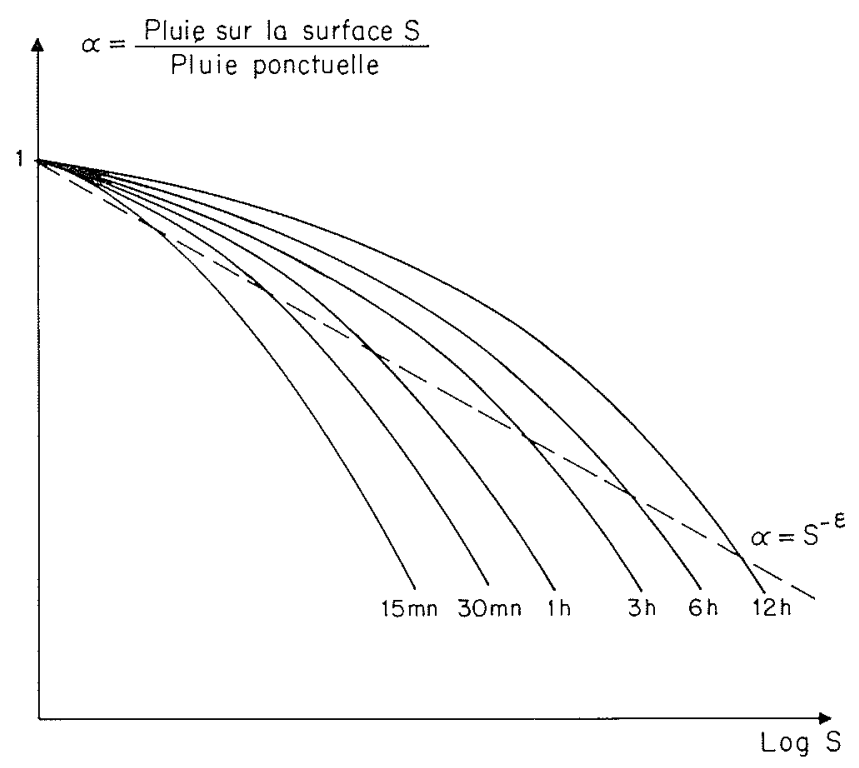

2/

\section{2 - Méthode " statistique "}

La voie statistique, que nous avions suivie, consistait à remettre en cause la définition de $t_{0}$ oar rien ne prouve qu'il faille bâtir un modèle du type de celui proposé par M. Caquot sur la notion de «durée de trajet de l'eau depuis le point extrême du bassin jusqu'à l'exutoire $»$. On peut cependant admettre que le modèle de Caquot puisse être basé sur un «temps caractéristique», lié aux paramètres physiques du bassin par une relation de la forme:

$$
t_{c}^{*}=\mu P^{c} S^{d} Q^{f}
$$

et faire un ajustement statistique global des constantes.

C'est ce que nous avons réalisé sur la base des résultats de mesures sur six bassins urbains expérimentaux de 1,5 à 30 ha, et des résultats d'un calcul hydraulique sur une aire urbaine de 200 ha.

On peut constater que, dans l'équation (4), les constantes $\mu$ et $(\beta+\delta)$ interviennent conjointement par le rapport :

$$
\frac{\mu^{\delta}}{\beta+\delta}
$$

( $b$ variant peu et restant compris en France entre - 0,4 et $-0,6)$.

Il en est de même pour $\varepsilon$ et $d$, dans l'exposant de $S$,

$$
(b d-\varepsilon+1)
$$

En fixant a priori $\varepsilon=0,015$ et $\mu=1$, l'ajustement conduit à :

$$
\begin{aligned}
& c=-0,40 \\
& d=0,43 \\
& f=0,27 \\
& \beta+\delta=0,96
\end{aligned}
$$

(SOGREAH)

Mais en fixant $\varepsilon=0,05$ on est conduit à $d=0,36$ $\varepsilon=0,1$ on est conduit à $d=0,26$

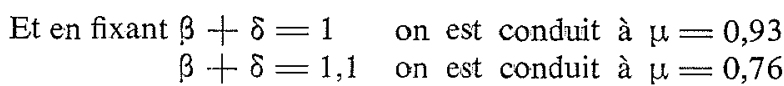

\section{3 - Confrontation des résultats}

Pour y voir un peu clair dans cette avalanche de valeurs, il est bon de recourir à l'arbitrage de résultats expérimentanx.

La confrontation doit cependant comporter deux aspects : - Adéquation «calcul-expérience » sur les bassins expérimentaux de 1,5 à 30 ha.

\begin{tabular}{|c|c|c|c|}
\hline \multicolumn{2}{|c|}{ Pour los bassins expérimentaux } & \multicolumn{2}{|c|}{ Pour la nódèle nathératique } \\
\hline $\begin{array}{l}a=0,96 \\
a=2,15 \\
a=3,26 \\
a=0,9, \\
a=2,10 \\
a=3,16\end{array}$ & $\begin{array}{l}b=0,39 \\
b=-0,46 \\
b=-0,51 \\
b=-0,37 \\
b=-0,40 \\
b=-0,43\end{array}$ & $\begin{array}{l}a=3,4 \\
a=3,9 \\
a=4,3\end{array}$ & $\begin{array}{l}\text { b. }-0,46 \\
b=-0,44 \\
b=0,40\end{array}$ \\
\hline
\end{tabular}

- Valeur d'une extrapolation possible au-delà de 30 ha.

La comparaison entre débits calculés et résultats expérimentaux est présentée dans le tableau 1.

Tableav 1

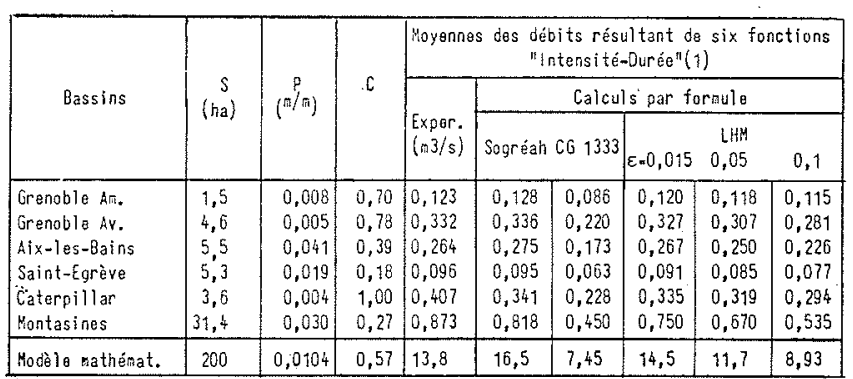

(1) Fonctions "Intensitémburéa": i a a tb

On peut évidemment constater le bon ajustement des constantes SOGREAH pour les bassins de 1,5 à 30 ha, puisque cette concordance constituait précisément le critère qui a permis de générer ces valeurs.

Par contre, cet ajustement conduit à une surestimation de $20 \%$ sur le calcul du modèle mathématique de 200 ha.

On peut aussi constater que :

- les valeurs de la CG 1333 conduisent à une sousestimation systématique dont la moyenne est de $39 \%$

- les valeurs proposées par le LHM conduisent à une sous-estimation moyenne de

$$
\begin{aligned}
6 \% \text { avec } \varepsilon & =0,015 \\
12 \% \text { avec } \varepsilon & =0,05 \\
19 \% \text { avec } \varepsilon & =0,1
\end{aligned}
$$

Pour résoudre la question de l'extrapolation qui devrait être autorisée dans la mesure où l'on peut encore parler d'une pente moyenne et d'un coefficient d'imperméabilisation 
moyen, il convient d'analyser la valeur de l'exposant de $S$ dans la formule de Caquot, qui pour $b=-0,5$ prend les valeurs suivantes:

\begin{tabular}{|c|c|c|}
\hline & \multicolumn{2}{|c|}{ valeur de $\frac{b d-\varepsilon+1}{1-b f}$} \\
\hline C6 1333 & & 0,80 \\
\hline LHW & $\begin{array}{l}\varepsilon=0,015 \\
\varepsilon=0,05 \\
\varepsilon=0,1\end{array}$ & $\begin{array}{l}0,85 \\
0,81 \\
0,75\end{array}$ \\
\hline SOGREAH & & 0,88 \\
\hline
\end{tabular}

Il est difficile de porter un jugement catégorique sur la comparaison de ces exposants, mais l'expérience acquise en hydrologie nous amène à considérer que l'exposant 0,88 , qui résulte de l'ajustement statistique jusqu'à 30 ha, est nettement trop fort pour une extrapolation jusqu'à 200 , 500 ou 1000 ha qui devrait être autorisée dans le cas de bassins versants homogènes. La valeur 0,80 doit constituer un ordre de grandeur raisonnable.

\section{4 - Essai de synthèse}

La confrontation présentée ci-dessus montre que l'approche conceptuelle intégrale du LHM avec $\varepsilon=0,05$ conduit à sous-estimer quelque peu les débits, mais doit permettre une extrapolation plus valable que notre ajustement statistique intégral.

Comme, en outre, les valeurs de $c$ et $f$, obtenues par les deux approches sont voisines, nous pensons que la synthèse devrait se faire sur l'ajustement de $\mu$. Ceci revient à admettre que le «temps oaractéristique», à prendre en compte dans le modèle de Caquot, est une fraction du «temps de concentration».

$$
t_{c}^{*}=k t_{c}=k \cdot 0,65 \cdot P^{-0,41} \cdot S^{0,507} \cdot Q^{-0,287}
$$

Les résultats de l'ajustement de $k$ sont indiqués dans le tableau 2.

La formule proposée actuellement serait donc:

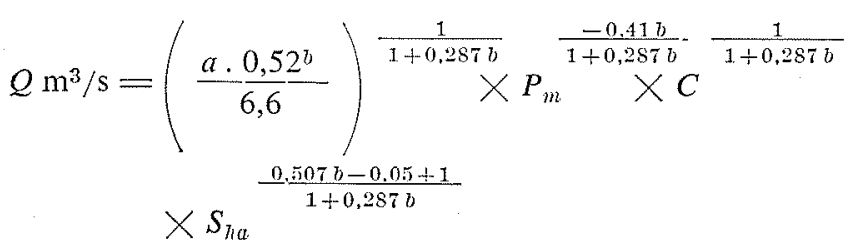

\begin{tabular}{|c|c|c|c|c|}
\hline \multirow{2}{*}{ Bassins } & \multirow{2}{*}{$\begin{array}{l}\text { Débits } \\
\text { axpérinentaux } \\
(\mathrm{a} 3 / \mathrm{s})\end{array}$} & \multicolumn{3}{|c|}{ Débits calculés par la formule LHK nodifléa } \\
\hline & & $\begin{array}{l}\varepsilon=0,015 \\
k=0,92 \\
\end{array}$ & $\begin{array}{l}\varepsilon=0,05 \\
k=0,80\end{array}$ & $\begin{array}{l}\varepsilon=0,1 \\
k=0,60\end{array}$ \\
\hline $\begin{array}{l}\text { Grenoble An. } \\
\text { Grenoble Av. } \\
\text { Aix-en-Provenco } \\
\text { SaintuEgrève } \\
\text { Caterofillar } \\
\text { Montasines }\end{array}$ & $\begin{array}{l}0,123 \\
0,332 \\
0,264 \\
0,090 \\
0,407 \\
0,873\end{array}$ & $\begin{array}{l}0,124 \\
0,338 \\
0,276 \\
0,094 \\
0,332 \\
0,777\end{array}$ & $\begin{array}{l}0,131 \\
0,342 \\
0,277 \\
0,094 \\
0,354 \\
0,726\end{array}$ & $\begin{array}{l}0,140 \\
0,347 \\
0,288 \\
0,095 \\
0,365 \\
0,660\end{array}$ \\
\hline $\begin{array}{l}\text { Modè lo } \\
\text { Yothétuatique }\end{array}$ & 13,8 & 14,9 & 13,0 & $10, \hat{0}$ \\
\hline
\end{tabular}

Tableau 2

L'incertitude moyenne par rapport aux résultats expérimentaux est $\pm 8 \%$. Cette incertitude reste valable jusqu'à 200 ha puisque la concordance est encore bonne avec les résultats du modèle mathématique qui n'a pas été utilisé dans l'ajustement de $k$.

Pour une extrapolation au-delà de 200 ha, on peut être tenté de faire reposer le poids de l'incertitude sur $\varepsilon$ :

- En prenant $\varepsilon=0,015$, conduisant à $k=0,92$, pour s'ajuster sur les données de 1 à 30 ha on est conduit, par rapport à la formule proposée, à des débits :

$$
\begin{aligned}
& \text { inférieurs de } 7 \% \text { pour } 1 \text { ha } \\
& \text { supérieurs de } 10 \% \text { pour } 100 \text { ha } \\
& \text { supérieurs de } 18 \% \text { pour } 1000 \text { ha }
\end{aligned}
$$

- En prenant $\varepsilon=0,1$ et $k=0,66$, on obtient des débits :

$$
\begin{aligned}
& \text { supérieurs de } 10 \% \text { pour } 1 \text { ha } \\
& \text { inférieurs de } 12 \% \text { pour } 100 \text { ha } \\
& \text { inférieurs de } 22 \% \text { pour } 1000 \text { ha }
\end{aligned}
$$

à ceux qui résulteraient de la formule proposée.

Les valeurs 0,1 et 0,015 constituent une «fourchette » extrême pour $\varepsilon$, et à notre avis le poids de $« d »$ dans l'exposant de $S$ est lui aussi très important pour assurer une extrapolation de la formule de Caquot aul-delà de 30 ha. Devant les difficultés pratiques et le coût des mesures directes qu'il serait alors nécessaire de réaliser sur des réseaux de 100 à 1000 ha, nous restons convaincus que c'est grâce aux modèles mathématiques d'écoulement que l'on pourra améliorer de façon déoisive l'analyse de l'exposant de $S$ dans la formule de Caquot. 


\section{Discussion}

Président : M. H. LORIFERNE

M. le Président remercie $M$. Normand de son intéressant exposé el ouvre la discussion.

M. LEMOINE (S.O.M.I.V.A.C.) intervient en ces termes :

J'ai eu l'occasion d'effectuer un travail, très proche de celui exposé par M. Normand, dans un essai d'adaptation à l'Afrique tropica'e de la formule de $M$. Caquot, à la demande du Comité Interafricain d'Etudes Hydrauliques ${ }^{(B)}$. En fait, une analyse détaillée de la formule de $\mathrm{M}$. CAQuoT montre qu'un certain nombre d'hypothèses simplificatrices, plus ou moins explicites, sont à la base de cette formule : hypothèse sur l'abattement, existence d'une relation $Q=f(A)$ liant le débit à la surface, hypothèse sur le tracé du réseau d'assainissement et sur lá forme du bassin. En fait, il serait plus logique de relier le temps de concentration à la longueur du bassin plutôt qu'à sa surface, et on a alors une formule à quatre paramètres au lieu de trois, ce qui, en fait, n'est pas plus complexe puisqu'on s'affranchit de la correction de forme du bassin proposée par M. CAQUOT.

M. Rousser signale quelques diflicultés rencontrées lors de l'application des formules du type de celles présentées par M. Normand aux cas concrets rencontrés en hydro'ogie urbaine.

Il serait intéressant, dit-il, de définir la méthode d'extrapolation de la formule de CAQuor corrigée, en particulier, en ce qui concerne : - la pente moyenne $P$;

- le coefficient de ruissellement $C$;

$1^{\circ}$ Pente moyenne :

Autant sa définition est simple pour une maille élémentaire, où l'acheminement de l'eau peut aisément être imaginé, autant elle est difficile dans une agglomération dense où les réseaux d'assainissement sont maillés et parfois à grande profondeur.

L'extrapolation sur $P$ pent-el'e éviter le passage par l'intermédiaire d'un modèle?

$2^{\circ}$ Coefficient de nissellement :

Comment relier $C$ aux paramètres de lurbanisation (coefficient doccupation des sols (COS), type d'urbanisme, conception des parkings, etc.)?

Inversement, comment prendre en compte dans les documents d'urbanisme, les contraintes d'assainissement? Certaines études ont-elles été engagées dans ce domaine?

M. Normand répond :

La formule de CaQvor, telle que nous l'avons ajustée, s'applique à un réseau ramifié et il est certain que, dans le cas d'un réseau maillé, les effets du stockage et de la propagation dans le réseau sur le débit maximal à l'exutoire seront différents. Il est alors nécessaire de décomposer le bassin total en bassins élémentaires et d'analyser, par un modele hydrodynamique, la composition et la propagation des hydrogrammes élémentaires dans le réseau maillé.

(a) Cf. Essai d'adaptation à l'Afrique tropicale des méthodes classigues de calcul du débit des ouvrages d'assainissement wban, par $\mathrm{L}$. L EXrorne et C. MicheL (Pub. du C.I.E.H.).
En outre, même avec un réseau ramifié, si le bassin présente des reliefs contrastés (une zone de forte pente et une zone de faible pente), la notion de pente moyenne $P$ perd sa signification car la formule de Caquot n'est pas linéaire en $P$, et il est souhaitable de décomposer, là aussi, le bassin total en bassins partiels et de faire appel à un modèle de propagation pour composer et propager les hydrogrammes partiels.

Pour ce qui concerne le coefficient de ruissellement, assimilé ici au taux d'imperméabilisation, il est souhaitable d'en effectuer l'estimation directe (par photos aériennes en fausse couleur, ou par une méthode de quadrillage) lorsque l'on étudie une zone urbaine déjà construite. Pour les projets d'urbanisation, l'idée de relier $C$ à des types d'occupation des sols a été retenue par la Commission que préside M. LORIFERNE.

M. le Président conclut la discussion en ces termes :

Comme vous l'avez vu, il est très important que, par des méthodes déterministes ou des méthodes statistiques, on puisse mieux déterminer les différents coefficients dont nous a parlé M. NoRMAND et arriver ainsi à améliorer les résultats donnés par la circulaire 1533 actuelle.

Pour répondre à la dernière question, posée par $M$. Rousser, le Groupe de travail qui a été constitué pour réviser cette circulaire, s'est, en effet, penché sur le problème du coefficient $C$; mais la question est encore en cours d'études.

Comme l'ont signalé d'ailleurs plusieurs orateurs, il faut arriver à améliorer la formule classique de $M$. CAQuor, mais il serait vain de chercher à obtenir ainsi une précision de l'ordre de quelques pour cent, car bien d'autres points sont entâchés d'incertitudes notables; par exemple, la période moyenne d'insuffisance (souvent décennale) admise pour le réseau, la valeur du coefficient $C$, etc. Il convient de faire en sorte, que les hypothèses admises sur chacun de ces points soient aussi proches que possible de la réalité, de façon à ne pas accumuler les erreurs; mais si on arrivait à déterminer le débit à $10 \%$ près, ce ne serait déjà pas mal, compte tenu de tontes les imprécisions encore inévitables.

$\mathrm{J}$ e vais maintenant demander à $\mathrm{M}$. RousseT de venir nous parler du modèle de gestion d'un réseau d'assainissement pluvial. M. RousSET s'est spécialement occupé de la Seine-Saint-Denis; ce département est assez particulier; très urbanisé, une grande partie de ses ouvrages d'assainissement sont déjà anciens; ils avaient été réalisés avant le rapide développement de l'urbanisation qui s'est produit depuis dix ou quinze ans; le réseau maillé soulevait de grandes diflicultés d'exploitation; mais il était, a priori, difficile de localiser les endroits où il y avait quelque chose à faire pour éviter les inondations qui, se produisent malheureusement chaque fois qu'il survient des orages importants.

Je donne la parole à $M$. Rousset.

M. Rousset présente la première partie de l'exposé qui décrit les objectifs recherchés par la mise sur pied d'un modèle mathématique. Ensuite. M. LORgeré (SOGREAH) expose, dans une deuxième partie, la construction et l'exploitation du modèle mathématique du réseau d'assainissement pluvial de la Seine-Saint-Denis ainsi que l'analyse des premiers résultats fournis par celui-ci. 